\title{
Fault localization in constraint programs
}

\author{
Nadjib Lazaar and Arnaud Gotlieb \\ INRIA Rennes Bretagne Atlantique, \\ Campus Beaulieu, 35042 \\ Rennes, France \\ Email: \{nadjib.lazaar, arnaud.gotlieb\}@inria.fr
}

\author{
Yahia Lebbah \\ Université d'Oran, Lab. LITIO, \\ 31000 Oran, Algeria \\ Université de Nice-Sophia \\ Antipolis, I3S-CNRS, France \\ Email: ylebbah@gmail.com
}

\begin{abstract}
-Constraint programs such as those written in highlevel modeling languages (e.g., OPL [15], ZINC [14], or COMET [16]) must be thoroughly verified before being used in applications. Detecting and localizing faults is therefore of great importance to lower the cost of the development of these constraint programs. In a previous work, we introduced a testing framework called CPTEST enabling automated test case generation for detecting non-conformities [13]. In this paper, we enhance this framework to introduce automatic fault localization in constraint programs. Our approach is based on constraint relaxation to identify the constraint that is responsible of a given fault. CPTEST is henceforth able to automatically localize faults in optimized OPL programs. We provide empirical evidence of the effectiveness of this approach on classical benchmark problems, namely Golomb rulers, n-queens, social golfer and car sequencing.
\end{abstract}

\section{INTRODUCTION}

These last years have seen the explosion of high-level constraint modeling languages, including OPL [15], COMET [16], ZINC [14], or Essence [7]. In parallel, several propositions were made to use constraint programs in critical applications. For example, constraint programs were developed for e-commerce [9], air-traffic control and management [6], [11] or critical software development [4], [8]. As any other programs, constraint programs written in high-level modeling languages must be thoroughly verified before being used on real-size instances of satisfaction or optimization problems. In [13], we introduced a testing framework where a first highly declarative constraint model is took as a reference to detect non-conformities within a refined and optimized constraint program solving the same problem. These non-conformities result from faults introduced during the refinement process, coming either from the absence or the bad formulation of constraints. Our testing framework was shown useful to detect non-conformities but it reveals itself poor at identifying the constraint responsible of the fault. In fact, the user resorts to explore manually one by one each of the constraints of its constraint model, which can be cumbersome even for small programs. For example, consider the $\mathrm{N}$-queens problem solved by the OPL program of Fig.1. When $N=30$, using a simple declarative model of N-queens, the testing framework of [13] reports a non-conformity: [30 $2928 \ldots 1$. Although this vector indeed solves all the constraints of Fig.1, it shows a fault in the OPL program which obviously places many queens on the same diagonal. Therefore, at least one constraint of the model

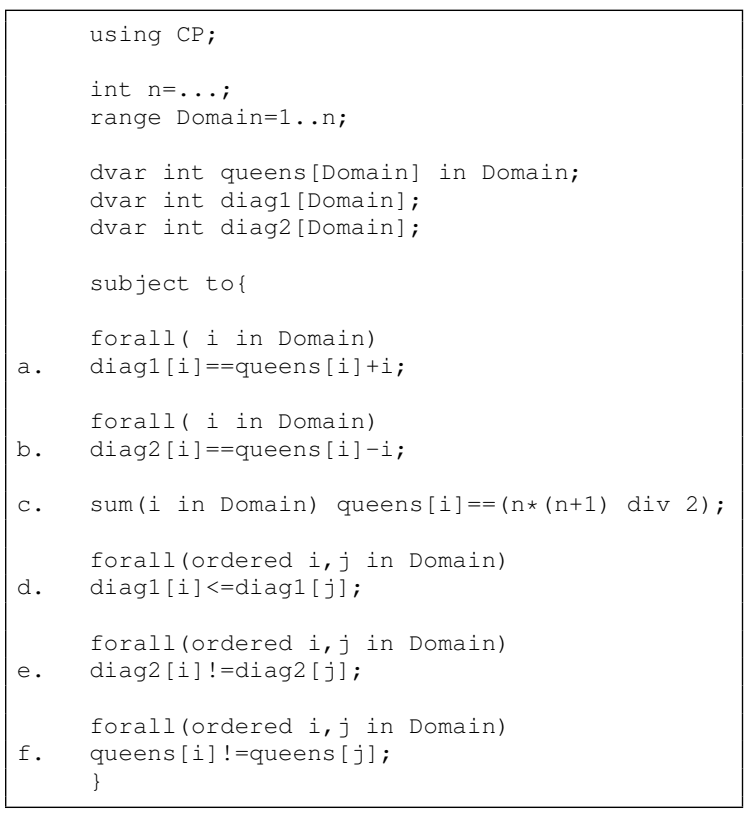

Fig. 1: N-queens problem in OPL.

is incorrectly formulated. In this paper, we address the problem of automatically locating the constraint that is responsible of such a non-conformity. Automatic fault localization is a difficult problem, even for conventional programs. The most successful approach in the Software Engineering community is the Jones, Statsko and Harrold's algorithm [10] that compares execution traces in order to guess the source code statements that are more likely to contain a fault. Their implementation called Tarantula takes both successful and unsuccessful execution traces of a sequential program as inputs, and by crossing these traces, it ranks the statements from the most suspicious to the less suspicious. The idea of the ranking algorithm is that faulty statements more frequently appear on unsuccessful traces. A related approach proposed by Cleve and Zeller aims at tracing back the causes of fault introduction [3], which is the ultimate goal of fault localization. Recently, it has been shown that the Jones, Statsko and Harrold's algorithm can be rephrased by using classical data mining indicators [1] leading to possible generalization to multi-faults localization. These approaches are well suited for sequential programs as the control flow is data-driven, i.e., any execution trace depends 
on the values of input variables. However, they are not suited for constraint programs as the control flow is constraint-driven in this case, i.e., the order on which constraints are considered by the language interpreter is usually not determined by input values. In other words, comparing traces on distinct instances does not help to localize faults. A trend in constraint program debugging has been to analyze a single trace via generic trace models [5], [12]. In the context of the OADymPPaC ${ }^{1}$ project, several post-mortem trace analyzers such as Codeine for Prolog and Morphine [12] for Mercury, ILOG Gentra4CP, and JPalm/JChoco have been developed. All these tools can help the developer to understand and optimize his constraint program, but they are not dedicated to automatic fault localization. Following the Shapiro's algorithmic debugging approach, the user still has to query the trace and user interaction during the debugging process is mandatory.

In this paper, we introduce a fault localization approach that is fully automated for OPL programs. This approach is based on systematic constraint relaxation by making use of the ILOG $\mathrm{CP}$ constraint solver. The idea is to solve auxiliary constraint programs built over the OPL constraint program under test and the reference model used to specify the problem. We provide an algorithm that takes the faulty constraint program and a non-conformity as inputs and returns either a single constraint or a set of constraints that are responsible of the fault. For example, in the OPL program of Fig.1, our algorithm reports constraint $\mathrm{d}$ as responsible of the given non-conformity. Looking at constraint $\mathrm{d}$ permits to spot that operator $=<$ should be replaced by $!=$. We present our implementation called CPTEST enabling automatic fault localization. We provide empirical evidence on classical benchmark problems, namely golomb rulers, n-queens, social golfer and car sequencing that our approach is not only applicable but also efficient to localize faults in OPL programs.

The rest of the paper is organized as follows: Section II gives an overview of the constraint programs testing framework of [13] and introduces some notations. Section III presents our automatic fault localization algorithm. Section IV presents CPTEST and the empirical results we got on n-queens, golomb rulers, social golfers and car sequencing. Section $\mathrm{V}$ concludes this paper and draws some perspectives to this work.

\section{BACKGROUND}

$\mathrm{CP}$ aims at solving satisfaction or optimization problems. When one faces a problem specification, one usually writes down a first declarative constraint model that faithfully represents the problem. We have high confidence in this model but playing with it rapidly shows that it cannot solve large instances of the problem. Then, this model is refined using classical techniques such as constraint reformulation, redundant and surrogate constraint addition, global constraint replacement, or static symmetry-breaking constraints. This refinement leads to have an optimized constraint model tuned

\footnotetext{
${ }^{1}$ http://contraintes.inria.fr/OADymPPaC/
}

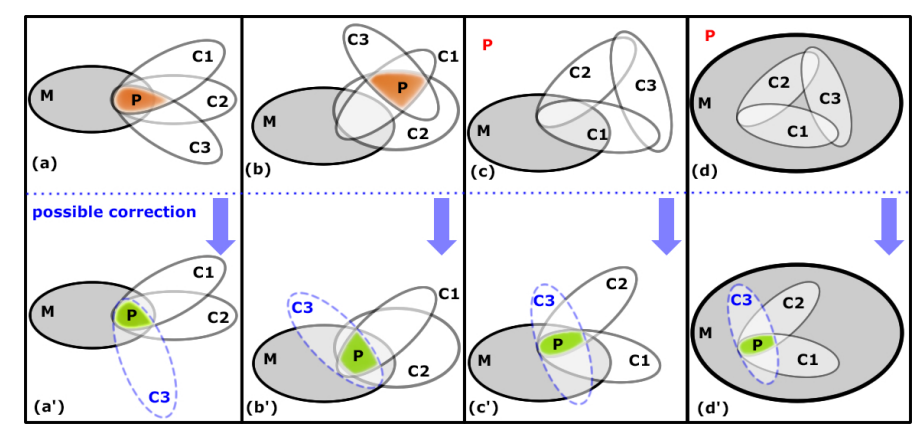

Fig. 2: Fault localization cases.

to address difficult instances. We think that most of the faults are introduced in this refinement step. In our previous work [13], we introduced a testing framework taking the first declarative model as an oracle for detecting non-conformities in the optimized model. We defined various formal conformity relations handling the cases where a single solution is found, all the solutions are found or a cost function has to be optimized.

\section{A. Notations}

In this section, we briefly give the necessary notations to understand the rest of the paper. $M$ denotes the initial declarative model called the Model-Oracle and $P$ denotes the Constraint Program Under Test (CPUT). Sol(M) (resp. $\operatorname{sol}(P)$ ) denotes the set of solutions of the model-oracle (resp. CPUT) where $M$ possesses at least one solution $(\operatorname{sol}(M) \neq \emptyset)$. Considering unsatisfiable model-oracles could be interesting for some applications but we excluded these cases in order to avoid considering equivalence of unsatisfiable models. The difference of two sets $E$ and $F$ is noted $E \backslash F \triangleq\{x /(x \in$ $E) \wedge(x \notin F)\}$. The general conformity relation between the model-oracle $M$ and the CPUT $P$ is given by the following definition:

\section{Definition 1 (conf):}

$$
P \operatorname{conf} M \Leftrightarrow \operatorname{sol}(P) \neq \emptyset \wedge \operatorname{sol}(P) \subseteq \operatorname{sol}(M)
$$

\section{B. Non-conformity detection}

As proving conformity on any instance of a problem is undecidable in the general case, we have proposed in [13], test algorithms aiming at detecting non-conformities. For a given instance, a non-conformity is either a solution of the CPUT $P$ that is not a solution of the model-oracle $M$, or $\mathrm{P}$ is reduced to fail (i.e., $\operatorname{sol}(P)=\emptyset$ ). Systematic non-conformities detection can be performed by combining the negation of a constraint of the model-oracle with the constraints of $P$ (i.e., $P \wedge \neg C_{i}$ where $\left.C_{i} \in M\right)$. Badly formulating a constraint in $P$ can remove and/or add solutions.

\section{FAUlt LOCALIZATION}

Once a non-conformity is detected, one faces the problem of localizing the faulty constraint. The major requirement on which our approach is based is the single fault hypothesis, 
i.e., there is only one constraint that is faulty in $P$. This requirement might appear as being restrictive but it has been shown that complex faults usually result from the coupling of single faults (a.k.a. the coupling effect [10]). In other words, faults that come from the interaction of several constraints are less likely to appear than single faulty constraint. Therefore, in our approach, when a non-conformity is found, we suppose that the CPUT $P$ misses a single constraint or has only one faulty constraint in it.

\section{A. Intuitions}

Fig. 2 depicts four distinct cases where the CPUT $P$ does not conform the model oracle $M(P \neg \operatorname{conf} M)$, i.e., there is a solution of $\mathrm{P}$ and not of $\mathrm{M}(\boldsymbol{p a r t}(\mathbf{a}, \mathbf{b}))$ or $\operatorname{sol}(P)=\emptyset$ (part (c, d)), where $\mathrm{P}$ is a conjunction of three constraints $C_{1} \wedge C_{2} \wedge C_{3}$. In Fig.2, $P$ is represented by the intersection of the three constraints and possible corrections are shown in blue.

- Part(a) exhibits a set of non-conformities (i.e., solutions of $P$ but not of $M$ ) where $P$ shares solutions with $\mathrm{M}$ (i.e., $\operatorname{sol}(M) \cap \operatorname{sol}(P) \neq \emptyset$ ). $\mathrm{P}$ is faulty as it contains $C_{3}$ a badly formulated constraint such that $\operatorname{sol}(P) \backslash \operatorname{sol}(M) \neq$ $\emptyset$. In fact, after having localized $C_{3}$, this model could be corrected as shown in $\operatorname{part}\left(\mathbf{a}^{\prime}\right)$.

- Part(b) exhibits another set of non-conformities where $\operatorname{sol}(P) \backslash \operatorname{sol}(M) \neq \emptyset$ and $\operatorname{sol}(M) \cap \operatorname{sol}(P)=\emptyset$. In this example, we have

$$
\left\{\begin{array}{l}
\operatorname{sol}(M) \cap \operatorname{sol}\left(C_{1}\right) \cap \operatorname{sol}\left(C_{2}\right) \neq \emptyset \\
\operatorname{sol}(M) \cap \operatorname{sol}\left(C_{1}\right) \cap \operatorname{sol}\left(C_{3}\right)=\emptyset \\
\operatorname{sol}(M) \cap \operatorname{sol}\left(C_{2}\right) \cap \operatorname{sol}\left(C_{3}\right)=\emptyset
\end{array}\right.
$$

hence, the faulty constraint can be localized as being $C_{3}$. Part(b') shows a possible correction of $P$ by revising $C_{3}$.

- Part(c) exhibits a case where $P$ is unsatisfiable. Here again, we have

$$
\left\{\begin{array}{l}
\operatorname{sol}(M) \cap \operatorname{sol}\left(C_{1}\right) \cap \operatorname{sol}\left(C_{2}\right) \neq \emptyset \\
\operatorname{sol}(M) \cap \operatorname{sol}\left(C_{1}\right) \cap \operatorname{sol}\left(C_{3}\right)=\emptyset \\
\operatorname{sol}(M) \cap \operatorname{sol}\left(C_{2}\right) \cap \operatorname{sol}\left(C_{3}\right)=\emptyset
\end{array}\right.
$$

hence $C_{3}$ is localized as the faulty constraint and should be revised as proposed in $\operatorname{part}\left(\mathbf{c}^{\prime}\right)$.

- Finally, Part(d) shows a case where $P$ is unsatisfiable but differs from the previous one as

$$
\left\{\begin{array}{l}
\operatorname{sol}(M) \cap \operatorname{sol}\left(C_{1}\right) \cap \operatorname{sol}\left(C_{2}\right) \neq \emptyset \Rightarrow C_{3} \text { may be faulty } \\
\operatorname{sol}(M) \cap \operatorname{sol}\left(C_{1}\right) \cap \operatorname{sol}\left(C_{3}\right) \neq \emptyset \Rightarrow C_{2} \text { may be faulty } \\
\operatorname{sol}(M) \cap \operatorname{sol}\left(C_{2}\right) \cap \operatorname{sol}\left(C_{3}\right) \neq \emptyset \Rightarrow C_{1} \text { may be faulty }
\end{array}\right.
$$

In this case, the faulty constraint cannot be localized and the set $\left\{C_{1}, C_{2}, C_{3}\right\}$ is returned as an overapproximation. In part(d'), we chose $C_{3}$ as a possible correction.

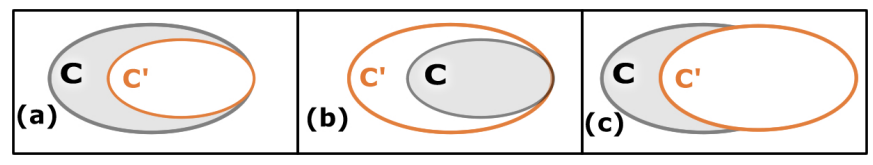

Fig. 3: Faulty constraint.

There are three distinct cases when considering nonconformities within $P$ w.r.t. $M$ : if $\mathrm{C}^{\prime}$ is a faulty version of a given constraint $\mathrm{C}$, then $\mathrm{C}^{\prime}$ ' can either removes actual solutions (such as in part(a) in Fig.3), either add "new solutions" (part(b) in Fig.3) or both (part(c) in Fig.3).

\section{B. The fault localization process}

Our fault localization process is based on the following definitions and property. Formally speaking, given a CPUT $P$ formed of $\left\{C_{1}, C_{2}, \ldots C_{n}\right\}$ constraints that are not conform w.r.t. its Model-Oracle $M$, then we introduce the notion of suspicious constraint as follows:

Definition 2 (Suspicious constraint):

$C_{i}$ is suspicious in $P$ w.r.t. $M$ $\equiv M \wedge\left\{C_{1}, C_{2} \ldots, C_{i-1}, C_{i+1} \ldots C_{n}\right\}$ is satisfiable.

$P$ does not conform $M$ iff either $P$ is unsatisfiable or there exists a non-conformity test case $n c$ such as $n c \in$ $\operatorname{sol}(P) \backslash \operatorname{sol}(M)$. Our fault localization process aims at reporting the set of suspicious constraints:

Definition 3 (SuspiciousSet): SuspiciousSet $(P) \equiv\{C \in$ $P: C$ is suspicious in $P$ w.r.t. $M$ \}

In the example of Fig.2, the fault localization process will return Suspicious $(P)=\left\{C_{1}, C_{2}, C_{3}\right\}$ for part(a) and $\operatorname{part}(\mathrm{d})$ as no one of these three constraints may be faulty while it will return singleton $\left\{C_{3}\right\}$ for both part(b) and part(c), showing that the faulty constraint is actually $C_{3}$. Note that, by construction, the set Suspicious $(P)$ is the smallest set containing all the suspicious constraints and then the overapproximation provided by this approach is also the more precise.

\section{Algorithm}

Based on the previous definitions, we propose an algorithm called locate that automatically localize suspicious constraints in $P$. Algo.1 takes as inputs the Model-Oracle $M$, the CPUT $P$ and a non-conformity $n c$ that has been automatically detected by other means [13]. The special case where $P$ is unsatisfiable and then there is no $n c$ is handled by a special tag. This algorithm returns SuspiciousSet $(P)$ noted $\operatorname{rev}\left(\left\{C_{1}, . ., C_{m}\right\}\right)$ that may be a singleton in the most favorable case or all the constraints of $P$ in the worst case. The algorithm returns also a second set addSet for the special case where all the constraints of $P$ are suspicious, and a nonconformity point $n c$ exists. In this case, $n c$ satisfies $P$, but does not satisfy some single constraint in $M$, according to the testing process [13]. The checking algorithm computes addSet, the subset of $M$ constraints that does not satisfy $n c$ : some of these constraints could be caught in $P$ in order to eliminate this non-conformity point. 


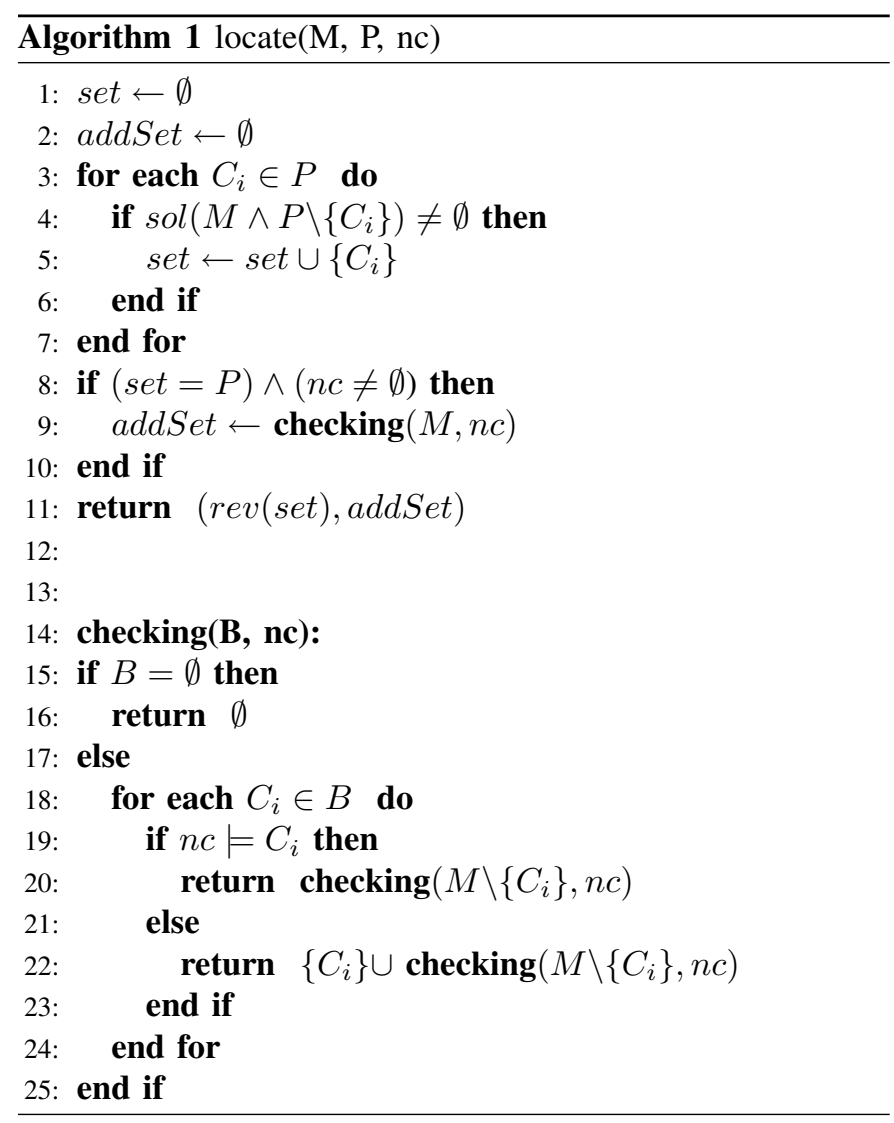

\section{Correctness and completeness}

Providing that the underlying constraint solver is correct and complete, Algo. 1 is correct as it will necessary return the faulty constraint within SuspiciousSet $(P)$. As $\operatorname{sol}\left(M \wedge P \backslash\left\{C_{i}\right\}\right) \neq$ $\emptyset$ is a sufficient condition for assessing that $C_{i}$ is actually a faulty constraint and Algo.1 loops on all the constraints, no suspicious constraint can be missed. But, this condition is not necessary as, by hypothesis, only a single constraint is faulty. Thus, algo. 1 is not complete and can return an over-approximation under the form of a set of suspicious constraints. Roughly speaking, algo.1 can return false alarms even in the case where a correct version of $P$ is submitted for fault localization. Note also that Algo.1 can return all the constraints of $P$ making the fault localization totally useless. Hopefully, this case is not frequent as it requires the conjunction of many unlikely events such as shown in Fig.4. In this example, there are three constraints where each pair of constraints shares common solutions.

\section{EXPERIMENTS}

All our experiments were performed on Intel Core2 Duo CPU 2.40Ghz machine with $2.00 \mathrm{~GB}$ of RAM and all the models we used to perform these experiments are available online at (www.irisa.fr/celtique/lazaar/CPTEST).

\section{A. CPTEST}

In this section, we give a brief overview of CPTEST, a first testing framework for constraint programs written on

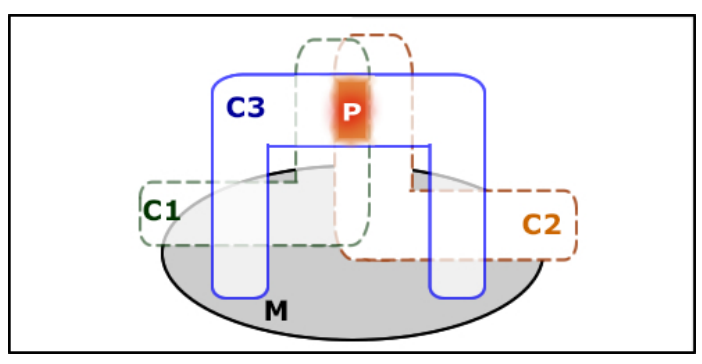

Fig. 4: Pathologic case

OPL. CPTEST includes a complete OPL parser and a backend process that produces dedicated OPL programs as output that have to be solved to detect and localize faults. The tool implements a process to detect fault and Algo.1 to localize this fault. CPTEST is based on ILOG CP optimizer 2.1.

The objective of our current experiment was to check that CPTEST can automatically detect and localize fault, using standard a fault injection process [2]. Then, we fed CPTEST with faulty CPUTs of four well-known CP problems, namely n-queens, social golfer, golomb rulers and car sequencing problems and we tried to localize faults by working on several instances from the easiest to the hardest.

\section{B. n-queens problem}

The problem of placing $n$ queens on an $n \times n$ chessboard is a classical problem where the model-oracle can be given by three constraints (noted m_ct $1, m \_c t 2$ and $m \_c t 3$ ), the first one insures that all queens are placed differently on their column, the second and the third constraint insure that no two queens are placed on the same upper or lower-diagonal on the chessboard. We improved this model by adding new data structures, redundant, surrogate and global constraints. We have produced 7 different faulty CPUTs (P1 to P7) ${ }^{2}$ for n-queens using fault injection.

Tab.I shows the results given by CPTEST on each faulty n-queens CPUT $(n=8)$. It shows the number of constraints and where fault was injected (e.g. p5_ct3 for P5), P6 and $\mathrm{P} 7$ are messing a part of $n$-queens specification where the fault injected respectively in $\mathrm{p} 6$ _ct 3 and $\mathrm{p} 7$ _ct 1 leads the CPUTs to accept new solutions. (part(b) Fig.3).

Tab.I shows also the non-conformity detected after the testing phase, the results of localization phase and its time consumption. P1, P2, P3 and P4 have no solution $(\operatorname{sol}(P i)=\emptyset)$ where the fault injected reduces them to fail. For instance let us take P3, the fault injected in is as follows:

p3_ct11: sum(i in Domain) queens $[i]==(n *(n+1) / 2)$; turned to

p3_ct11: sum(i in Domain) queens [i] $==(n+(n+1) / 2)$;

${ }^{2} \mathrm{We}$ recall that all models and CPUTs are available on (www.irisa.fr/celtique/lazaar/CPTEST). 
TABLE I: Fault detection and localization of n-queens problem

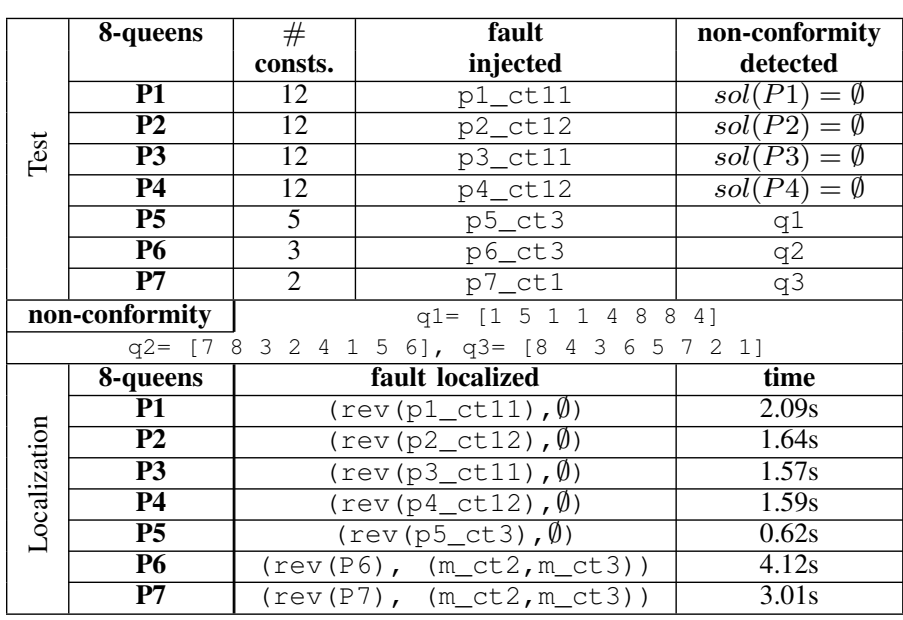

CPTEST returns (rev(p3_ct11), $\emptyset)$, here the set of suspicious constraint is the singleton p3_ct11 the faulty constraint that has to be revised. The faults are localized for the four first programs in few seconds by pointing each time the faulty constraint that has to be revised.

The fault injected in P5 leads it to have bad n-queens solutions, testing phase highlights this non-conformity ([1 $\left.\left.\begin{array}{lllllll}5 & 1 & 1 & 4 & 8 & 8 & 4\end{array}\right]\right)$. For localization phase, CPTEST points the faulty constraint in less than a second and returns p5_ct3 to be revised.

For P6 and P7 the fault is that, respectively, p6_ct 3 and p7_ct 1 do not catch a part of n-queens specification. The two CPUTs accept consequently new solutions. CPTEST detects non-conformities that are bad solutions revealing faults in P6 and P7. For localization, let us take P6, CPTEST returns ( $r e v(P 6),\left(m \_c t 2, m \_c t 3\right)$ ), in less than five seconds. The set of suspicious constraint is here equals to P6, CPTEST returns also a set of $\mathrm{M}$ constraints (m_ct2, m_ct 3 ) that should be considered in P6.

\section{Social golfer problem}

Social golfer is one of the famous problems in the CSPLib (prob010). We have $m$ social golfers, $n$ weeks and $k$ groups of $l$ size. Each golfer plays once a week in groups of $l$ golfers. The problem is to build a schedule of play for all golfers over the $n$ weeks such that no golfer plays in the same group as any other golfer more than one time.

The model-oracle can be built by two constraints ( m_ct1 and $m_{\text {_ct }} 2$ ), the first one to specify that each group has exactly $l$ golfers (group size). The second one to say that each pair of golfers meets only at most once.

The model-oracle can after be improved using static symmetry breaking. The symmetry can be broken by selective assignment. We get a model with five constraints, we inject
TABLE II: Fault detection and localization of social golfer problem

\begin{tabular}{|c|c|c|c|c|}
\hline \multirow{6}{*}{$\overrightarrow{\overrightarrow{0}}$} & $\begin{array}{l}\text { Social } \\
\text { golfer }\end{array}$ & $\begin{array}{c}\# \\
\text { constrs. }\end{array}$ & $\begin{array}{c}\text { fault } \\
\text { injected }\end{array}$ & $\begin{array}{c}\text { non-conformity } \\
\text { detected }\end{array}$ \\
\hline & P1 & 5 & p1_ct 1 & $\operatorname{sol}(P 1)=\emptyset$ \\
\hline & $\mathbf{P 2}$ & 5 & p2_ct2 & nc1 \\
\hline & $\mathbf{P 3}$ & 5 & p3_ct3 & $\operatorname{sol}(P 3)=\emptyset$ \\
\hline & $\mathbf{P 4}$ & 5 & p4_ct 4 & $\operatorname{sol}(P 4)=\emptyset$ \\
\hline & P5 & 5 & p5_ct5 & $\operatorname{sol}(P 5)=\emptyset$ \\
\hline \multicolumn{2}{|c|}{ non-conformity } & \multicolumn{3}{|c|}{$\mathrm{nc1}=\left[\left[\begin{array}{llll}1 & 1 & 1 & 1\end{array}\right]\left[\begin{array}{llll}1 & 2 & 2 & 2\end{array}\right]\left[\begin{array}{llll}1 & 3 & 3 & 3\end{array}\right]\right.$} \\
\hline & $\left.\begin{array}{lll}1 & 3 & 3\end{array}\right]\left[\begin{array}{ll}2 & 2\end{array}\right.$ & 1] $\left[\begin{array}{ll}2 & 2\end{array}\right.$ & $\left.\begin{array}{lll}3 & 3 & 2\end{array}\right]\left[\begin{array}{ll}3 & 1\end{array}\right.$ & 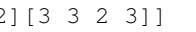 \\
\hline \multirow{6}{*}{ 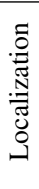 } & social golfer & \multicolumn{2}{|c|}{ fault localized } & time \\
\hline & P1 & \multicolumn{2}{|c|}{$\left(\right.$ rev $\left.\left(\mathrm{p} 1 \_c t 1\right), \emptyset\right)$} & $1.04 \mathrm{~s}$ \\
\hline & $\mathbf{P 2}$ & \multicolumn{2}{|c|}{$\left(\operatorname{rev}(\mathrm{P} 2), \quad\left(\mathrm{m} \_\mathrm{ct} 2\right)\right)$} & $0.18 \mathrm{~s}$ \\
\hline & $\mathbf{P 3}$ & \multicolumn{2}{|c|}{$\left(r e v\left(p 3 \_c t 3\right), \emptyset\right)$} & $1.05 \mathrm{~s}$ \\
\hline & $\overline{\mathbf{P 4}}$ & \multicolumn{2}{|c|}{$\left(\operatorname{rev}\left(\mathrm{p}^{4} \_\mathrm{ct} 4, \mathrm{p}^{4} \_\mathrm{ct} 5\right), \emptyset\right)$} & $1.03 \mathrm{~s}$ \\
\hline & P5 & \multicolumn{2}{|c|}{$\left(\operatorname{rev}\left(\mathrm{p} 5 \_\mathrm{ct} 5\right), \emptyset\right)$} & $1.15 \mathrm{~s}$ \\
\hline
\end{tabular}

in each constraint a fault to get at the end five faulty CPUTs (P1 to P5). We launch CPTEST on these faulty CPUTs to first detect fault and after localize them. We take an instance of the problem with 9 golfers ( 3 groups of 3 golfers) playing during 4 weeks.

Tab.II shows the results given by CPTEST on each faulty CPUT. The fault injected in P1, P3 and P5 are respectively on p1_ct 1, p3_ct3 and p5_ct5. These faults reduce the solution space to empty for the three CPUTs, so there are non-conform to the model-oracle. For instance, let us take P5, the fault in p5_ct 5 is:

p5_ct5: forall (g in 1.golfers, w in 1..weeks:
\[ \mathrm{w}>=2 \& \& g<=\text { groupsize) } \] assign $[\mathrm{g}, \mathrm{w}]==\mathrm{g}$;

turned to

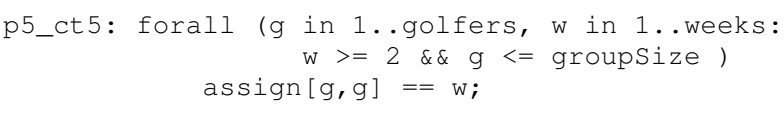

CPTEST localizes the fault (in $\simeq 1 s$ ) and returns the singleton p5_ct 5 the constraint to be revised.

The fault injected in p2_ct2 leads P2 to accept new solutions. In this case, P2 can return bad solutions. CPTEST detects one of them by testing, nc1 is a non-conformity (a bad solution of social golfer) where golfer 3 and golfer 4 play in the same group (group3) over 2 weeks ( $3 r d$ and 4 th week). CPTEST returns (rev(P2), (m_ct2)) that the developer has to reformulate a constraint in P2 to catch (m_ct2) the second constraint of the model-oracle.

P4 is a case where CPTEST returns a set of suspicious constraint. The fault is injected in p4_ct 4 and CPTEST returns (p4_ct 4, p4_ct5) an over-approximation of constraints. The two constraints in P4 break symmetries. Let us imagine that the search space $S R$ can be divided in two symmetric sets: $S R \equiv S_{1} \cup S_{2}$ where p4_ct 5 removes $S_{2}$. p4_ct 4 divides $S_{1}$ in two symmetric sets $\left(S_{1} \equiv S_{3} \cup S_{4}\right)$ and removes $S_{4}$. By adding the two constraints, the search space is reduced to $S_{3}$. The fault injected to $\mathrm{p} 4$ _ct 4 removes $S_{1}$ where $\mathrm{p} 4$ _ct 5 
TABLE III: Fault detection and localization of Golomb rulers problem
TABLE IV: Fault detection and localization of car sequencing problem

\begin{tabular}{|c|c|c|c|c|c|c|c|c|}
\hline \multirow{8}{*}{$\stackrel{\vec{\omega}}{\oplus}$} & $\begin{array}{l}\text { Golomb } \\
\text { rulers }\end{array}$ & $\begin{array}{c}\# \\
\text { consts }\end{array}$ & $\begin{array}{c}\text { fault } \\
\text { injected }\end{array}$ & $\begin{array}{c}\text { non-conformity } \\
\text { detected }\end{array}$ & \multirow{8}{*}{$\stackrel{\vec{\vartheta}}{\oplus}$} & $\begin{array}{c}\text { car } \\
\text { sequencing }\end{array}$ & $\begin{array}{c}\text { fault } \\
\text { injected }\end{array}$ & $\begin{array}{c}\text { non-conformity } \\
\text { detected }\end{array}$ \\
\hline & P1 & 2 & $\mathrm{p} 1$ _ct 2 & {$\left[\begin{array}{llll}0 & 1 & 3 & 6\end{array}\right]$} & & P1 & p1_ct2 & slots 1 \\
\hline & $\mathbf{P 2}$ & 4 & p2_ct 3 & {$\left[\begin{array}{llll}0 & 1 & 6 & 11\end{array}\right]$} & & $\mathbf{P 2}$ & p2_ct 3 & slots2 \\
\hline & $\mathbf{P 3}$ & 5 & p3_ct 3 & {$\left[\begin{array}{llll}0 & 5 & 6 & 11\end{array}\right]$} & & $\mathbf{P 3}$ & p3_ct 2 & slots3 \\
\hline & P4 & 9 & p4_ct 5 & $\operatorname{sol}(P 4)=\emptyset$ & & P4 & p4_ct 2 & $\operatorname{sol}(P 4)=\emptyset$ \\
\hline & P5 & 9 & p5_ct 1 & $\operatorname{sol}(P 5)=\emptyset$ & & P5 & p5_ct 1 & $\operatorname{sol}(P 5)=\emptyset$ \\
\hline & P6 & 9 & p6_ct 2 & {$\left[\begin{array}{llll}0 & 1 & 6 & 11\end{array}\right]$} & & P6 & p6_ct 6 & $\operatorname{sol}(P 6)=\emptyset$ \\
\hline & P7 & 9 & p7_ct 9 & $\operatorname{sol}(P 7)=\emptyset$ & & P7 & p7_ct 5 & $\operatorname{sol}(P 7)=\emptyset$ \\
\hline \multirow{8}{*}{ 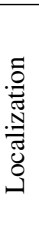 } & Golomb rulers & \multicolumn{2}{|c|}{ fault localized } & time & & & \multirow{3}{*}{\multicolumn{2}{|c|}{$\begin{array}{l}\text { slots } 1=\left[\begin{array}{llllllllll}4 & 5 & 3 & 6 & 4 & 6 & 5 & 1 & 3 & 2\end{array}\right] \\
\operatorname{slots} 2=\left[\begin{array}{llllllllll}4 & 6 & 3 & 1 & 5 & 2 & 3 & 5 & 4 & 6\end{array}\right] \\
\operatorname{slots} 3=\left[\begin{array}{llllllllll}5 & 2 & 3 & 6 & 1 & 4 & 3 & 6 & 4 & 5\end{array}\right]\end{array}$}} \\
\hline & P1 & (re & $\left.\left(m \_c t 2\right)\right)$ & $0.32 \mathrm{~s}$ & & & & \\
\hline & $\mathbf{P 2}$ & (re & $\left.\left(m \_c t 2\right)\right)$ & $0.82 \mathrm{~s}$ & & & & \\
\hline & P3 & (re & $\left.\left(m \_c t 2\right)\right)$ & $1.15 \mathrm{~s}$ & \multirow{8}{*}{ } & cSeq & \begin{tabular}{|c|} 
fault localized \\
\end{tabular} & time \\
\hline & $\mathbf{P 4}$ & \multicolumn{2}{|c|}{$\left(r e v\left(p 4 \_c t 5\right), \emptyset\right)$} & $1.87 \mathrm{~s}$ & & P1 & $(\operatorname{rev}(\mathrm{P} 1),($ m_ct 2$))$ & $0.46 \mathrm{~s}$ \\
\hline & $\mathbf{P 5}$ & \multicolumn{2}{|c|}{$\left(r e v\left(p 5 \_c t 1\right), \emptyset\right)$} & $1.62 \mathrm{~s}$ & & $\mathbf{P 2}$ & $(\operatorname{rev}(\mathrm{P} 2), \quad($ m_ct 2$))$ & $0.23 \mathrm{~s}$ \\
\hline & $\mathbf{P 6}$ & \multicolumn{2}{|c|}{$(\operatorname{rev}(\mathrm{P} 6), \quad($ m_ct 2$))$} & $2.14 \mathrm{~s}$ & & $\mathbf{P 3}$ & $(\operatorname{rev}(\mathrm{P} 3), \quad($ m_ct 2$))$ & $0.67 \mathrm{~s}$ \\
\hline & $\mathbf{P 7}$ & $(r e v)$ &, $\mathrm{p} 7 \_$ct 9$),(0)$ & $1.92 \mathrm{~s}$ & & $\mathbf{P 4}$ & $\left(r e v\left(p 4 \_c t 2\right), \emptyset\right)$ & $0.87 \mathrm{~s}$ \\
\hline & & & & & & P5 & $\left(\operatorname{rev}\left(\mathrm{p} 5 \_\mathrm{ct} 1\right), \emptyset\right)$ & $1.29 \mathrm{~s}$ \\
\hline & & & & & & $\mathbf{P 6}$ & $\left(r e v\left(p 6 \_c t 6\right), \emptyset\right)$ & $1.34 \mathrm{~s}$ \\
\hline & & & & & & $\mathbf{P 7}$ & $\left(r e v\left(p 7 \_c t 5, p 7 \_c t 6\right), \emptyset\right)$ & $1.28 \mathrm{~s}$ \\
\hline
\end{tabular}

removes $S_{2}$, in this case the search space is reduced to empty and either p4_ct 4 or p4_ct 5 can be revised.

\section{Golomb rulers}

A Golomb ruler is a set of $m$ marks $\left(0=x_{1}<x_{2} \ldots<x_{m}\right)$ where the $m(m-1) / 2$ distances $\left\{x_{j}-x_{i} \mid 1 \leq i<j \leq m\right\}$ are distinct. A ruler is of order $m$ and its length is $x_{m}$. The objective is to find an optimal ruler (minimal length) of order $m$.

A first declarative model (model-oracle) includes two constraints. m_ct 1 to order the marks and m_ct 2 to have different distances between these marks.

We improve this model using different techniques, the use of new data structure (e.g. d for distances), the addition of a global constraint (allDifferent (d)), the addition of surrogate and redundant constraints. We also break symmetry by adding specific constraints. We obtain at the end 7 faulty CPUT by fault injection.

We take a small instance of Golomb rulers $(m=4)$. We launch CPTEST on these faulty CPUTs where Tab.III shows the results. The three first CPUTs accept new solutions where the fault injected is of type part(b) Fig.3. For each of these three CPUTs a non-conformity is reached. For example, if we take P2, CPTEST returns after testing [ $\left[\begin{array}{llll}0 & 1 & 6 & 11\end{array}\right]$ that is a bad Golomb ruler where $6-1=11-6$. CPTEST returns after localization ( rev (P2), (m_ct2)), here P2 has to catch the second constraint of the model-oracle by reformulating one of its constraints.

P4 and P5 are also non-conform to the model-oracle where $\operatorname{sol}(\mathrm{P} i)=\emptyset$. The faults are respectively in $\mathrm{p} 4$ _ct 5 and p5_ct 1 . Let us take P4 to show the injected fault:

p4_ct5: allDifferent(all(ind in indexes ) d[ind]); turned to

p4_ct5: forall(i in $m . .2 * m)$

count (all (j in indexes) $d[j], i)==1$;
CPTEST returns a singleton as suspecious constraints set for P4 and P5 (resp. p4_ct5 and p5_ct 1 ) in a reasonable time consumption.

P6 have a bad formulation on p6_ct 2 and CPTEST returns a non-conformity. For localization, CPTEST returns m_ct 2 to be caught in P6.

On P7, we injected one fault at p7_ct $9(\neq$ turned to $=)$. This fault reduces $\operatorname{sol}(P 7)$ to empty. CPTEST returns two suspicious constraints ( 7 7 ct 6 and p7_ct 9). This is due to the interference created by the injected fault between the faulty $\mathrm{p} 7$ _ct 9 and $\mathrm{p} 7$ _ct 6 where the two constraints are dedicated to a static symmetry breaking.

\section{E. Car sequencing}

Car sequencing is an interesting $\mathrm{CP}$ problem that tries to find an assembly line of cars for a car-production company where cars are grouped on classes. Each class represents cars with some specific options. The assembly line must satisfy some option capacity constraints.

The model-oracle of car sequencing is taken from the OPL book [15]. The model-oracle is composed of two constraints (m_ct 1 and m_ct2). The first one expresses demand constraints for how many cars of a given class have to be produced. The second one expresses capacity constraints of the form 1 outof $u$ for: Each sub-sequence of $u$ cars, a unit can produce at most 1 cars with a given option.

We improve the original model and we follow the same approach as for the precedent problems by injecting faults. We have at the end 7 faulty CPUTs.

We took an instance of the problem with an assembly line of 10 cars, 6 classes and 5 options. Tab.IV shows the results of CPTEST for fault detection and localization on car sequencing problem.

The three first CPUTs (P1, P2 and P3) have the same characteristics that accept new solutions and are non-conform 
to the model-oracle, the faults are injected respectively in p1_ct2, p2_ct3 and p3_ct2. CPTEST returns a nonconformity point for the three first CPUTs (resp. slots1, slots 2 and slots3) that are bad assembly lines. To localize faults, CPTEST returns all constraints as an overapproximation. It proposes a model-oracle constraint m_ct 2 to be caught in the CPUTs.

P4, P5 and P6 have a faulty constraint (resp. p4_ct2, p5_ct 1 and p6_ct 6). The faults injected in these CPUTs reduce the set of solutions to empty and CPTEST detects this non-conformity.

If we take P5, the fault injected is a bad formulation as:

p5_ct1: forall(i in Cars) sum(j in slots)

$((\operatorname{slot}[j]==i) *($ one $[j]))==\operatorname{demandV}[i]$;

turned to

p5_ct1: pack(slot, slot, one);

This constraint is returned by CPTEST ( (rev ( ct 1$), \emptyset)$ in $1.29 s$.

In P7, the fault is injected in $\mathrm{p} 7$ _ct 5 where P7 is nonconform to the model-oracle. CPTEST returns in localization a set of suspicious constraints (p7_ct5, p7_ct 6).

\section{CONCLUSion}

In this paper, we introduced a novel approach for automatic fault localization in constraint programs. According to our knowledge, this is the first time an approach that is not based on post-mortem trace analysis is proposed: the advantage being that our approach does not require any user interaction. We enhanced CPTEST, our CP testing framework for OPL programs, with automatic fault localization. And we got empirical evidence that shows our approach is efficient to localize fault in OPL programs. The experimental evaluation was performed on classical benchmarks such as Golomb rulers, n-queens, social golfers and car sequencing. In many cases, CPTEST can localize exactly the constraint responsible of a reported non-conformity efficiently. In a few cases, several constraints are proposed among which the faulty constraint is necessarily included. However, our approach is currently built on the hypothesis that a single constraint is responsible of the nonconformity which is a limitation. Further work includes the removal of this limitation as well as the extension of CPTEST to automatic correction of faulty constraints.

\section{REFERENCES}

[1] Peggy Cellier, Mireille Ducassé, Sébastien Ferré, and Olivier Ridoux. Dellis: A data mining process for fault localization. In Proc. of the 21 st International Conference on Software Engineering \& Knowledge Engineering (SEKE'2009), pages 432-437, 2009.

[2] Jeffrey A. Clark and Dhiraj K. Pradhan. Fault injection. Computer, 28(6):47-56, 1995.

[3] Holger Cleve and Andreas Zeller. Locating causes of program failures. In ICSE '05: Proceedings of the 27th international conference on Software engineering, pages 342-351, New York, NY, USA, May 2005.

[4] H. Collavizza, M. Rueher, and P. Van Hentenryck. Cpbpv: A constraintprogramming framework for bounded program verification. In Proc. of CP2008, LNCS 5202, pages 327-341, 2008
[5] P. Deransart, M. V. Hermenegildo, and J. Maluszynski, editors. Analysis and Visualization Tools for Constraint Programming, Constrain Debugging (DiSCiPl project), volume 1870 of Lecture Notes in Computer Science. Springer, 2000.

[6] P. Flener, J. Pearson, M. Agren, Garcia-Avello C., M. Celiktin, and S. Dissing. Air-traffic complexity resolution in multi-sector planning. Journal of Air Transport Management, 13(6):323 - 328, 2007.

[7] A.M. Frisch, M. Grum, C. Jefferson, B. Martnez, and H.I. Miguel. The design of essence: a constraint language for specifying combinatorial problems. In Proceedings of IJCAI'07, pages 80-87, 2007.

[8] A. Gotlieb. Tcas software verification using constraint programming. The Knowledge Engineering Review, 2009. Accepted for publication.

[9] Alan Holland and Barry O'Sullivan. Robust solutions for combinatorial auctions. In ACM Conference on Electronic Commerce (EC-2005), pages 183-192, 2005.

[10] J. A. Jones, M. J. Harrold, and J. Stasko. Visualization of test information to assist fault localization. In ICSE '02: Proceedings of the 24th International Conference on Software Engineering, pages 467477, 2002.

[11] U. Junker and D. Vidal. Air traffic flow management with ilog $\mathrm{cp}$ optimizer. In International Workshop on Constraint Programming for Air Traffic Control and Management, 2008. 7th EuroControl Innovative Research Workshop and Exhibition (INO'08).

[12] L. Langevine, P. Deransart, M. Ducassé, and E. Jahier. Prototyping clp(fd) tracers: a trace model and an experimental validation environment. In WLPE, 2001.

[13] N. Lazaar, A. Gotlieb, and Y. Lebbah. On testing constraint programs. In Proc. of Principles of Constraint Programming, CP'2010, Sept. 2010.

[14] K. Marriott, N. Nethercote, R. Rafeh, P. J. Stuckey, M. Garcia De $\mathrm{La}$ Banda, and $\mathrm{M}$. Wallace. The design of the zinc modelling language. Constraints, 13(3):229-267, 2008.

[15] P. Van Hentenryck. The OPL optimization programming language. MIT Press, Cambridge, MA, USA, 1999.

[16] Pascal Van Hentenryck and Laurent Michel. Constraint-Based Local Search. The MIT Press, 2005. 